\title{
Eficacia y seguridad del cierre quirúrgico del conducto arterioso permeable por el cirujano pediatra general: ensayo clínico
}

\author{
Effectiveness and safety of the surgical closure of permeable arteriosus conduct by the \\ general pediatric surgeon: clinical trial
}

\author{
Antonio F. Gallardo-Meza', José M. González-Sánchez', Francisco Vidrio-Patrón', \\ Irene L. Velarde-Briceño ${ }^{1}$, Alejandra Peña-Juárez², Humberto Murguía-Guerrero', \\ María Teresa Martínez-González³, Oscar E. Ceja-Mejía ${ }^{4}$, Miguel A. Medina-Andrade, \\ Paulina Armas-Quiroż ${ }^{4}$ Brenda N. Arias-Uribe ${ }^{4}$, Elizabeth López-Villalobos ${ }^{4 *}$ y Humberto Vázquez-Jackson ${ }^{1}$ \\ ${ }^{1}$ Departamento de Cirugía Pediátrica; ${ }^{2}$ Departamento de Cardiología Pediátrica; ${ }^{3}$ Departamento de Pediatría; ${ }^{4}$ Unidad de Cuidados Intensivos \\ Neonatales. Hospital General de Occidente, Hospital Santa María Chapalita, Guadalajara, Jalisco, México
}

\begin{abstract}
Resumen
Antecedentes: El conducto arterioso permeable (CAP) es un defecto cardiaco congénito y se considera un problema de salud pública. Se presenta en un alto porcentaje de recién nacidos y en algunos mayores de 1 mes. El cierre farmacológico es el tratamiento inicial preferido, ya que ha tenido excelentes resultados; sin embargo, en aquellos casos en los que no es posible, está indicado el cierre quirúrgico. Objetivo: Evaluar la eficacia y la seguridad del cierre quirúrgico del CAP por cirujanos pediatras sin especialidad en cirugía cardiovascular. Método: Ensayo clínico realizado en pacientes del Hospital General de Occidente, centro hospitalario público de segundo nivel, con diagnóstico de CAP, que requirieron corrección quirúrgica. Se revisaron en forma retrospectiva los expedientes de enero de 2001 a diciembre de 2018. Resultados: Se incluyeron 224 pacientes divididos en dos grupos: grupo I, con 184 (82\%) recién nacidos, y grupo II, con 40 (18\%) niños grandes de 2 meses a 8 años de edad. A todos se les realizó cierre quirúrgico: 3 por toracoscopía y 221 por toracotomía posterolateral izquierda. Presentaron complicaciones 36 pacientes, lo que representa el $16 \%$ del total; solo el $5.3 \%$ fueron complicaciones mayores. Fallecieron 24 pacientes en el posoperatorio, lo que representa una mortalidad del 10.7\%; ninguno falleció por complicaciones transquirúrgicas. El CAP es un defecto cardíaco congénito que se presenta en alto porcentaje en pacientes prematuros. El cierre farmacológico es el principal tratamiento por tener excelentes resultados en recién nacidos; sin embargo, en aquellos casos en los que no sea posible está indicado el cierre quirúrgico. Todos los pacientes fueron operados por cirujanos pediatras generales, con una sobrevida global del 92\%. Conclusiones: En los hospitales donde no hay cirujano cardiovascular pediátrico ni cardiólogo intervencionista, la corrección quirúrgica del CAP puede ser llevada a cabo por un cirujano pediatra. La técnica es reproducible, fácil de realizar y con mínimas complicaciones.
\end{abstract}

Palabras clave: Conducto arterioso permeable. Recién nacido prematuro. Tratamiento quirúrgico.

Correspondencia:

*Elizabeth López Villalobos

E-mail: lizylovi@ hotmail.com
Fecha de recepción: 22-01-2020

Fecha de aceptación: 21-08-2020

DOI: 10.24875/ACM.20000014
Disponible en internet: 23-02-2021

Arch Cardiol Mex. 2021:91(1):73-83

www.archivoscardiologia.com

1405-9940 / @ 2020 Instituto Nacional de Cardiología Ignacio Chávez. Publicado por Permanyer. Este es un artículo open access bajo la licencia CC BY-NC-ND (http://creativecommons.org/licenses/by-nc-nd/4.0/). 


\section{Abstract}

Background: The Patent Ductus Arteriosus (PDA) is congenital heart defect and is considered a public health problem. It occurs in a high percentage of newborns and in some older than 1 month. Pharmacological closure is the preferred initial treatment, as it has had excellent results; however, in those cases where it is not possible, surgical closure is indicated. Objective: The objective is to evaluate the efficacy and safety of the surgical closure of the patent PDA when it is carried out by pediatric surgeons without specialization in cardiovascular surgery. Methods: This study was conducted at the West General Hospital, a 2nd level public hospital, with the diagnosis of patent ductus arteriosus that required surgical correction. For the collection of the information, the files from January 2001 to December 2018 were retrospectively reviewed. Results: 224 patients were included; divided into two groups: Group I: 184 (82\%) "newborns" and Group II: 40 (18\%) "big children" with ages from 2 months to 8 years. All had a surgical closure; 3 by thoracoscopy and 221 by left posterolateral thoracotomy. 36 patients presented complications representing $16 \%$ of the total of patients, only $5.3 \%$ were major complications. 24 patients died in the postoperative period, representing a mortality of $10.7 \%$, none died due to trans-surgical complications. PDA is a congenital heart defect that occurs in a high percentage of premature patients. The pharmacological closure is the principal treatment because it has had excellent results in newborns; however, in those cases where it is not possible, surgical closure it's indicated. All patients were operated by general pediatric surgeons, with a global survival of 92\%. Conclusions: We conclude that in hospitals where there is no pediatric cardiovascular surgeon or interventional cardiologist, the surgical correction of the PDA can be carried out by a general pediatric surgeon. The technique is reproducible, easy to perform and with minimal complications.

Key words: Ductus arteriosus. Premature birth. Surgical treatment.

\section{Introducción}

El conducto arterioso permeable (CAP) es la cardiopatía congénita que se presenta con mayor frecuencia en niños recién nacidos (RN) prematuros. Es el más común de los defectos cardiacos congénitos ${ }^{1-4}$ y afecta a uno de cada $5,000 \mathrm{RN}$ a término ${ }^{5}$. La incidencia global en RN prematuros es del $50-70 \%$, llegando a afectar hasta al $80 \%$ de los RN pretérmino de muy bajo peso $(<1000 \mathrm{~g})^{6-8}$. Está íntimamente relacionado con la morbimortalidad de los RN prematuros, por lo cual se considera un importante problema de salud pública.

Los RN con más alto riesgo de presentar CAP son los que desarrollan síndrome de dificultad respiratoria (SDR), taquipnea transitoria del RN (TTRN), neumonía intrauterina, síndrome de aspiración de meconio o hipertensión pulmonar persistente del $\mathrm{RN}^{6-8}$. El diagnóstico solo con datos clínicos es difícil, ya que los síntomas y signos se pueden confundir con algunos problemas respiratorios del $\mathrm{RN}$ o con septicemia, lo cual hace que el diagnóstico de un CAP hemodinámicamente significativo (CAP-HP) sea tardío.

Las unidades de cuidados intensivos neonatales cada vez tienen mayor éxito en la sobrevida de los pacientes prematuros. Esto se debe a que el diagnóstico no invasivo de cardiopatías con equipos portátiles de ecocardiografía Doppler es de fácil acceso a dichas unidades. Por ende, el diagnóstico temprano de CAP se ha incrementado de manera importante.
Un diámetro de CAP > $1.5 \mathrm{~mm}$, con una relación flujo pulmonar/flujo sistémico $>1.5$, es un índice altamente predictivo de que el CAP no se va a cerrar y de que, por lo tanto, un alto porcentaje de estos casos desarrollarán un CAP-HP, lo que permite tomar decisiones tempranas para el tratamiento. En la actualidad, el tratamiento de elección es el cierre farmacológico, con buenos resultados en diferentes estudios. Sin embargo, en caso de que este cierre no se logre, se puede realizar el cierre percutáneo mediante cateterismo por parte de un cardiólogo intervencionista, o el cierre quirúrgico con toracotomía por parte de un cirujano pediatra cardiovascular $^{9-15}$.

En este estudio mostramos nuestros resultados con el cierre quirúrgico del CAP en aquellos pacientes que no respondieron al cierre farmacológico o que tenían alguna contraindicación para este.

No existen publicaciones en las que se comparen las tasas de éxito o fracaso en el cierre del CAP realizado por un cirujano pediatra general en comparación con un cirujano pediatra cardiovascular ${ }^{16}$.

El objetivo de este trabajo es demostrar que el cierre quirúrgico del CAP puede llevarse a cabo con éxito por cirujanos pediatras sin especialidad en cirugía cardiovascular, lo cual facilita el manejo de la cardiopatía congénita más frecuente. El motivo es la falta de un servicio de cirugía cardiovascular pediátrica o de intervencionismo en los hospitales de segundo y tercer 
nivel en México, así como en muchos países semejantes al nuestro.

\section{Método}

Estudio descriptivo en el cual se analizó a pacientes con CAP, con especial atención en el cierre quirúrgico y su evolución, complicaciones y mortalidad. Se realizó en el Hospital General de Occidente, un centro hospitalario público de segundo nivel que presta atención de salud a la población pediátrica abierta.

Los criterios de inclusión fueron tener diagnóstico de CAP que requiriera corrección quirúrgica. Se excluyeron del estudio los pacientes sin expediente completo. Para la recolección de la información se revisaron en forma retrospectiva los expedientes de enero de 2001 a diciembre de 2018, que supusieron un total de 227 cierres quirúrgicos de CAP. Se encontraron 224 expedientes completos; el resto, tres pacientes, no tenían el expediente completo o ya estaba depurado, por lo cual fueron excluidos.

Las variables revisadas fueron el sexo, la edad gestacional, el motivo de ingreso, el tratamiento farmacológico, la edad y el peso en el momento de la cirugía, el tipo de abordaje, el tiempo quirúrgico, la valoración de hipertensión pulmonar, la imposibilidad para extubar al paciente, el tiempo de extubación tras la cirugía, las complicaciones y la mortalidad.

De la población estudiada, se dividió a los pacientes en dos grupos (Fig. 1):

- Grupo I: recién nacidos de 24.1 hasta 41 semanas de edad gestacional.

- Grupo II: niños grandes, que incluía pacientes con edades desde 2 meses hasta 8 años.

Los pacientes del grupo I ingresaron a la unidad de cuidados intensivos neonatal por prematuridad y algún problema asociado: respiratorio (SDR o TTRN), sepsis neonatal, hipoxia neonatal, desnutrición asimétrica u otros. Los pacientes del grupo II ingresaron por datos clínicos de repercusión hemodinámica, falla para crecer o infecciones respiratorias frecuentes, y durante su abordaje diagnóstico se encontró el CAP.

Los pacientes que entraban en el protocolo de cierre eran aquellos con datos clínicos y ecocardiográficos de CAP-HP. Los datos clínicos eran dependencia de oxígeno, deterioro respiratorio, imposibilidad de extubación, requerir aumento en los parámetros de ventilación mecánica, descompensación hemodinámica e hipoperfusión de órganos diana (insuficiencia renal aguda o enterocolitis necrotizante). Los datos en el ecocardiograma Doppler que en el momento del estudio se

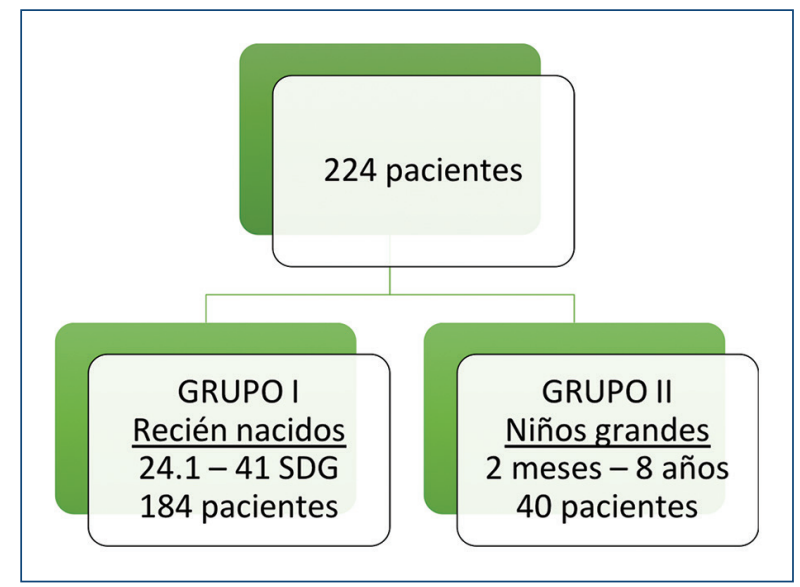

Figura 1. Distribución por edades. SDG: semanas de gestación.

tomaron en cuenta fueron la presencia de un conducto con un diámetro $>1.5 \mathrm{~mm}$ y una relación flujo pulmonar/ flujo sistémico $>1.5$. Debe considerarse también la relación aurícula izquierda/aorta $>1.5$ con datos añadidos como el robo diastólico en la aorta descendente.

Los pacientes dentro del protocolo de cierre se dividieron en dos grupos:

- Grupo A: pacientes sometidos inicialmente a cierre farmacológico con inhibidores de la ciclooxigenasa o prostaglandinas (o ambos); si no había respuesta ecocardiográfica, los pacientes se sometían a cierre quirúrgico.

- Grupo B: pacientes con alguna contraindicación para el cierre farmacológico que pasaban directamente a cierre quirúrgico.

La técnica quirúrgica utilizada en los pacientes de ambos grupos fue por toracotomía posterolateral izquierda, con abordaje extrapleural inicialmente y en caso de desgarro de la pleura se completa intrapleural. Se identifica el conducto teniendo como referencia primordial el nervio vago y el laríngeo recurrente, se diseca con cuidado en todo su diámetro y se hace doble ligadura con seda 3-0 o 4-0 sin sección del conducto en los pacientes del grupo I, y con sección y sutura en los pacientes del grupo II. Con el abordaje extrapleural no se dejó tubo pleural; con el intrapleural, se dejó un tubo pleural por 24 horas.

En los casos toracoscópicos el abordaje es con tres puertos de $3 \mathrm{~mm}$ : línea clavicular posterior, quinto espacio intercostal, por el cual se introduce la óptica, y bajo visión directa se introducen otros dos puertos, uno en la línea clavicular posterior séptimo u octavo espacio intercostal, y el otro en la línea clavicular media 


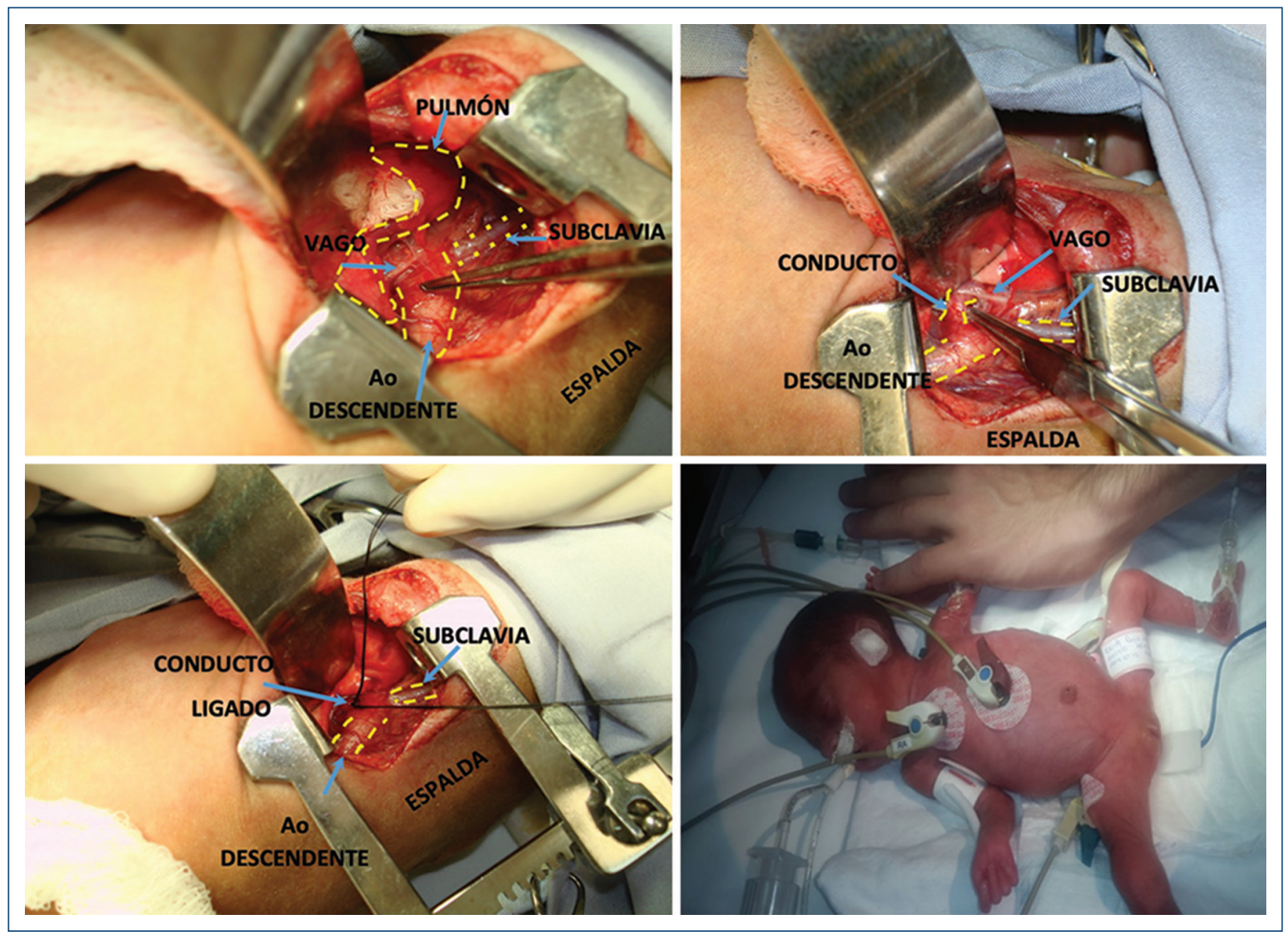

Figura 2. Fotos de la técnica quirúrgica. Ao: aorta.

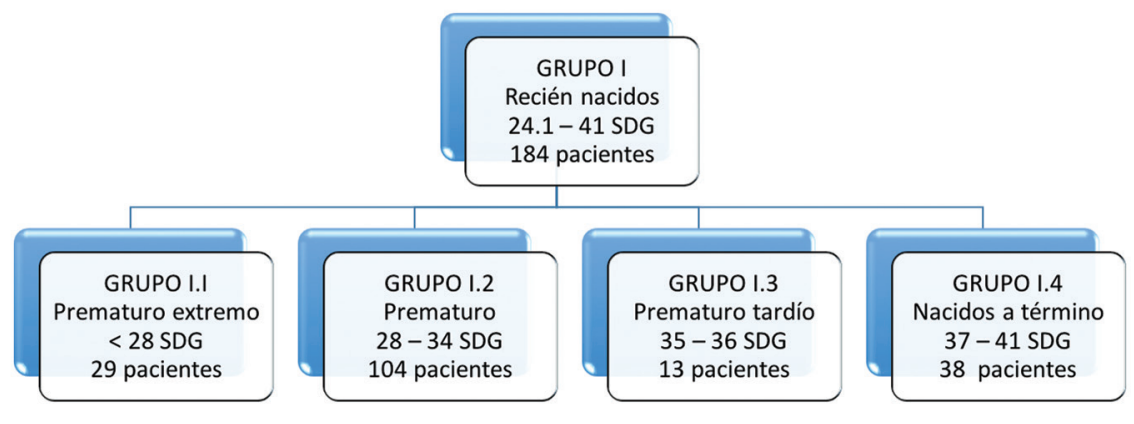

Figura 3. Distribución por edades de los pacientes del grupo I. SDG: semanas de gestación.

séptimo espacio intercostal. Se hace un abordaje intrapleural y el conducto se liga con una grapa Haemolock de $5 \mathrm{~mm}$, dejando un tubo intrapleural por 24 horas (Fig. 2).

Se obtuvo la aprobación del procedimiento quirúrgico por medio de consentimiento informado, firmado por los representantes legales de los pacientes.

\section{Resultados}

De los 227 pacientes operados, se incluyeron 224 con expedientes completos y se dividieron en dos grupos (I y II) según la edad. En el grupo I hubo 184 pacientes (Fig. 3). El motivo de ingreso a la unidad de cuidados intensivos neonatal de los pacientes del 


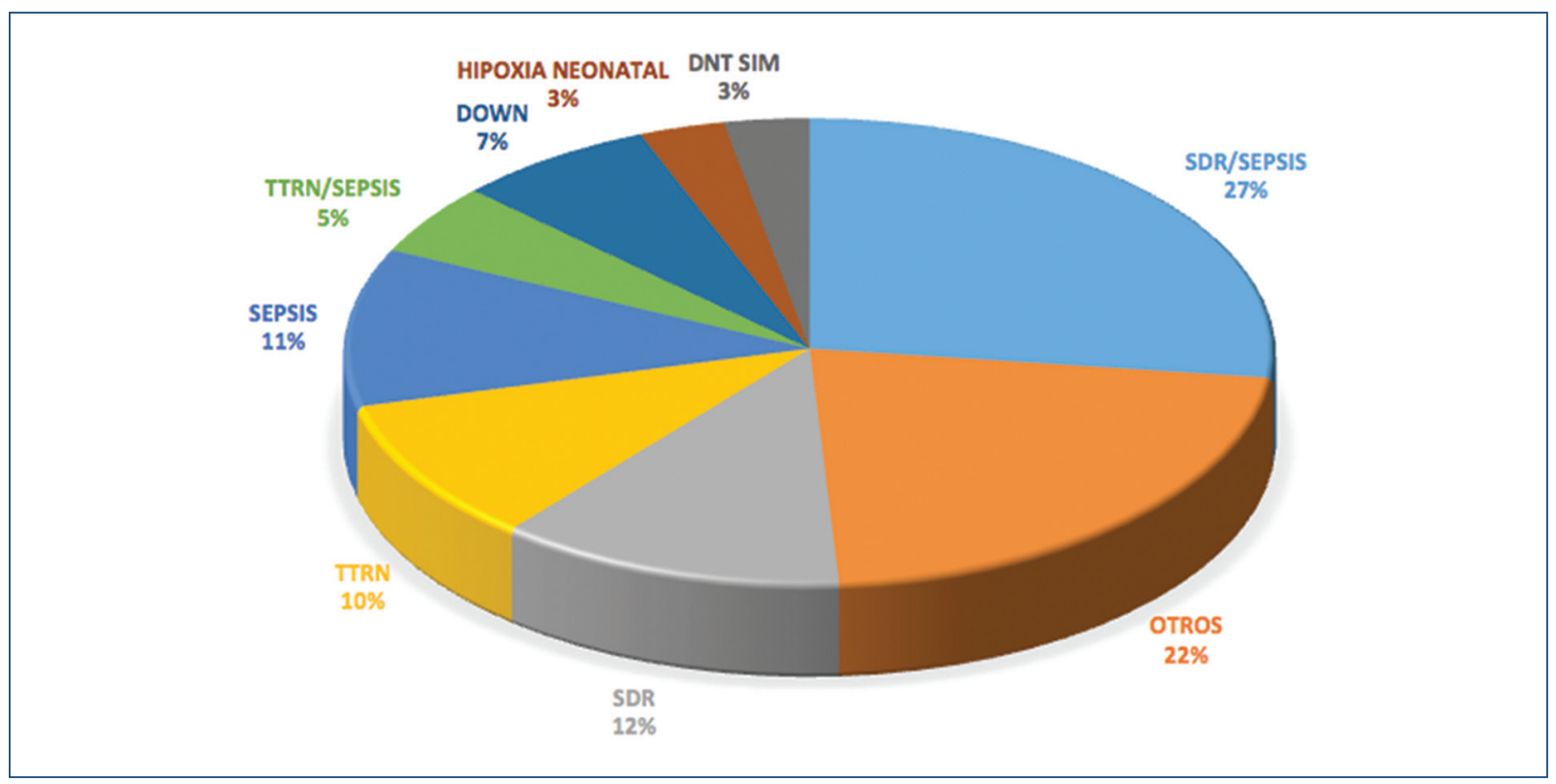

Figura 4. Diagnóstico de ingreso en los pacientes del grupo I. DNT SIM: desnutrición simétrica; SDR: síndrome de dificultad respiratoria; TTRN: taquipnea transitoria del recién nacido.

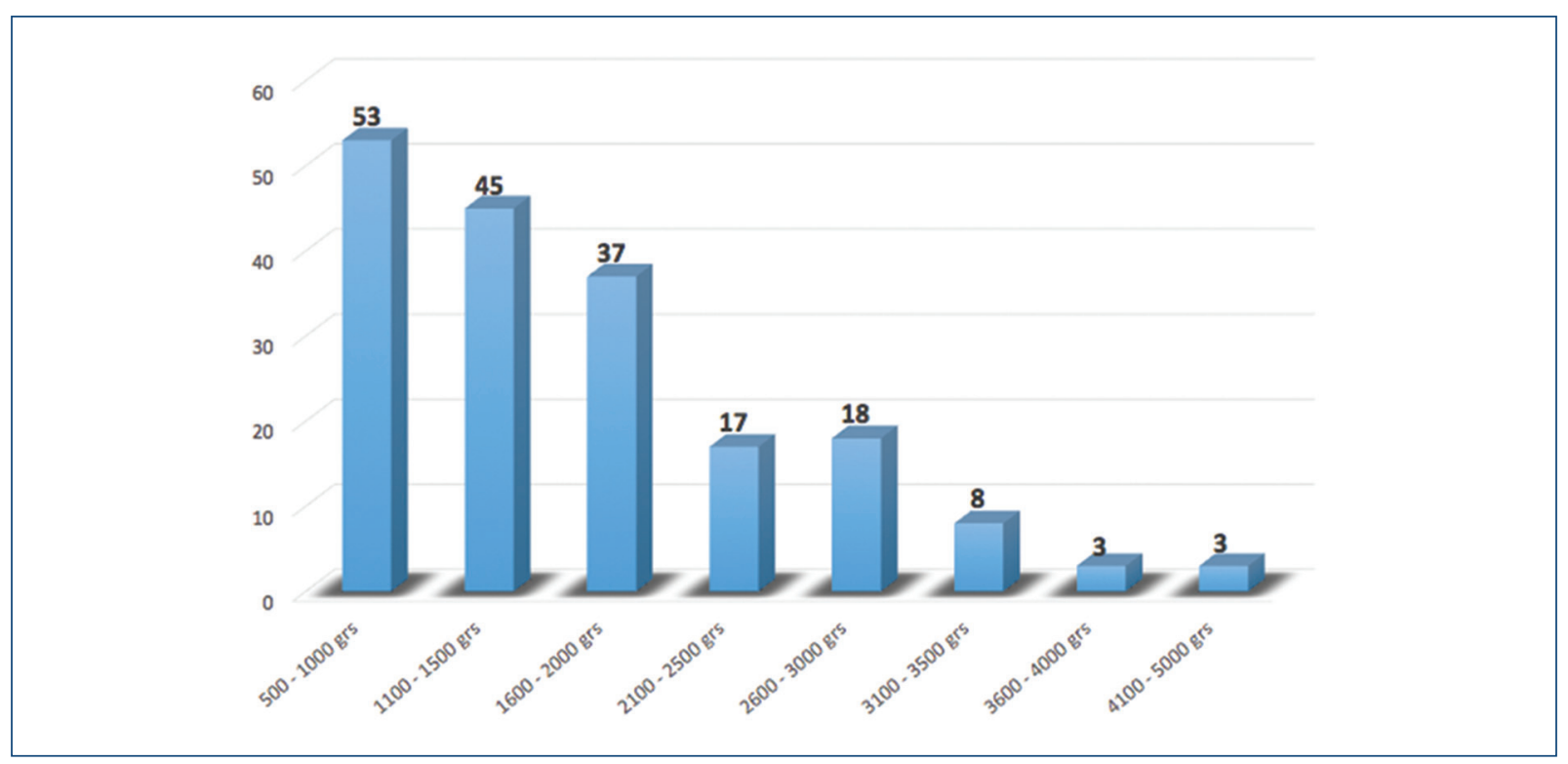

Figura 5. Distribución de peso (de $500 \mathrm{~g}$ a $5 \mathrm{~kg}$ ) en los pacientes del grupo l.

grupo I fue por prematuridad y algún problema asociado, encontrando que el más frecuente era SDR asociado a sepsis en 49 pacientes (27\%). Los diagnósticos incluidos en otros problemas fueron síndrome dismórfico, producto macrosómico, parto fortuito, hipoglucemia, ictericia por incompatibilidad de grupo ABO, policitemia, hernia diafragmática izquierda, atresia duodenal y malformación anorrectal (Fig. 4).
El peso de los pacientes varió entre $500 \mathrm{~g}$ y $5 \mathrm{~kg}$ (Fig. 5), y en cuanto al sexo, fueron 81 (44\%) niñas y $103(56 \%)$ niños.

Con respecto al cierre farmacológico, se dividieron en dos grupos (Fig. 6):

- Grupo A: 86 pacientes (46\%) se sometieron a cierre farmacológico a base de paracetamol, ibuprofeno, ketoprofeno o indometacina, con un primer ciclo de 


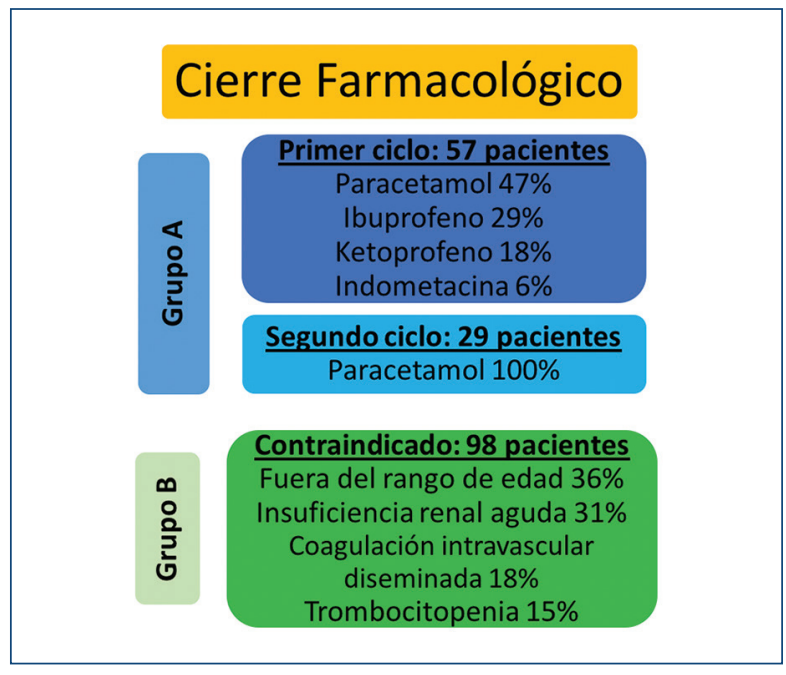

Figura 6. Tratamiento farmacológico.

3 días. En caso de no haber respuesta ecocardiográfica se intentó un segundo ciclo de 3 días con paracetamol en 29 pacientes, finalmente sin lograr el cierre, por lo que fueron sometidos a cirugía.

- Grupo B: 98 pacientes (54\%) tuvieron alguna contraindicación para el cierre farmacológico y pasaron directamente a cierre quirúrgico.

Tenían algún grado de hipertensión pulmonar 102 (56\%) pacientes, de los cuales en 32 (31\%) era leve, en $65(63 \%)$ era moderada y en $5(6 \%)$ era grave. Cursaron sin hipertensión arterial pulmonar $82(44 \%)$ pacientes.

De los 184 pacientes de nuestra casuística del grupo I, 19 (10\%) tenían cardiopatía asociada: 8 (4\%) comunicación interventricular, 5 (3\%) comunicación interauricular, $4(2 \%)$ pseudocoartación aórtica pero sin gradiente significativo, y 2 (1\%) canal auriculoventricular (uno de estos últimos tuvo que ser operado del conducto para ofrecerle mayor tiempo de vida y poder ser operado en una institución de tercer nivel).

En los pacientes del grupo I se reporta un tiempo quirúrgico de 30 a 45 minutos en el $5 \%$ de los pacientes y de 45 minutos a 1 hora en el $28 \%$. El abordaje extrapleural fue el preferido y se realizó en 141 pacientes (77\%).

Los pacientes con CAP cursan con imposibilidad para la extubación por la congestión pulmonar secundaria al aumento del flujo pulmonar que produce el conducto; por lo tanto, el tiempo de extubación tras la cirugía se tomó como un parámetro de éxito del tratamiento quirúrgico, y encontramos que la mayoría de los pacientes $(59 ; 32 \%)$ fueron extubados en los primeros 5 días, $26(14 \%)$ a los $6-10$ días, $10(5.5 \%)$ a los $11-15$ días, $3(1.5 \%)$ a los $16-20$ días y $8(4.5 \%)$ tras más de
20 días. No se logró extubar a 23 pacientes (12.5\%) por presentar complicaciones graves inherentes a su prematuridad y sus diagnósticos de ingreso, y fallecieron. El resto, 55 pacientes (30\%), se extubaron en quirófano (Fig. 7).

Las complicaciones del grupo I estuvieron presentes en 33 pacientes, que representan el $18 \%$ de los RN. Solo el $6.5 \%$ tuvieron complicaciones mayores secundarias a los cambios hemodinámicos posteriores al cierre del conducto: 11 pacientes (29\%) con neumotórax que se resolvió con la colocación de una sonda pleural; $4(12 \%)$ con isquemia intestinal no grave que se resolvió con reposo intestinal y antibioticoterapia; 3 $(9 \%)$ con edema agudo pulmonar manejado con restricción de líquidos y diurético; 3 (9\%) con hemorragia pulmonar; $3(9 \%)$ con choque cardiogénico manejado con aminas; 2 (6\%) con quilotórax manejados con sonda pleural, ayuno, nutrición parenteral total con aporte de triglicéridos de cadena media y octreotida; 2 (6\%) con parálisis diafragmática que se corrigieron, cuando sus condiciones lo permitieron, por medio de plicatura diafragmática; 2 (6\%) con infección o dehiscencia de herida quirúrgica que se resolvieron con curaciones; 2 $(6 \%)$ con fuga residual del conducto observada en el ecocardiograma de control; y 1 (3\%) con desgarro del conducto que se reparó en el momento sin repercusión hemodinámica (Fig. 8).

Fallecieron 23 pacientes (12.5\%), y las principales causas de defunción fueron: 6 (26\%) por falla orgánica múltiple, 4 (17\%) por choque séptico, 3 (13\%) por secuelas neurológicas debidas a hemorragia interventricular, $3(13 \%)$ por hemorragia pulmonar, $2(9 \%)$ por edema agudo pulmonar, 2 (9\%) por choque cardiogénico, 1 (4\%) por quilotórax, 1 (4\%) por coagulación intravascular diseminada y 1 (4\%) por insuficiencia renal aguda. Ninguna de las defunciones sucedió en el transoperatorio y cabe mencionar que las causas de muerte eran inherentes a la prematuridad.

En la figura 9 se muestra la distribución por edades en el grupo II. Los motivos de ingreso en el grupo II fueron: 12 pacientes con neumonías de repetición, 9 con soplo en estudio, 6 con síndrome de Down, 6 con cianosis en estudio y 5 con falla para crecer. En estos pacientes, durante su abordaje diagnóstico el ecocardiograma reportó el CAP. Hubo dos pacientes con síndrome de Marfan y síndrome de Alagille que tenían CAP y fueron ingresados para su corrección quirúrgica (Fig. 10).

En el grupo II, el peso de los pacientes varió entre 3 y $19 \mathrm{~kg}$ (Fig. 11). En cuanto al sexo, fueron 23 (57\%) niñas y $17(43 \%)$ niños. 


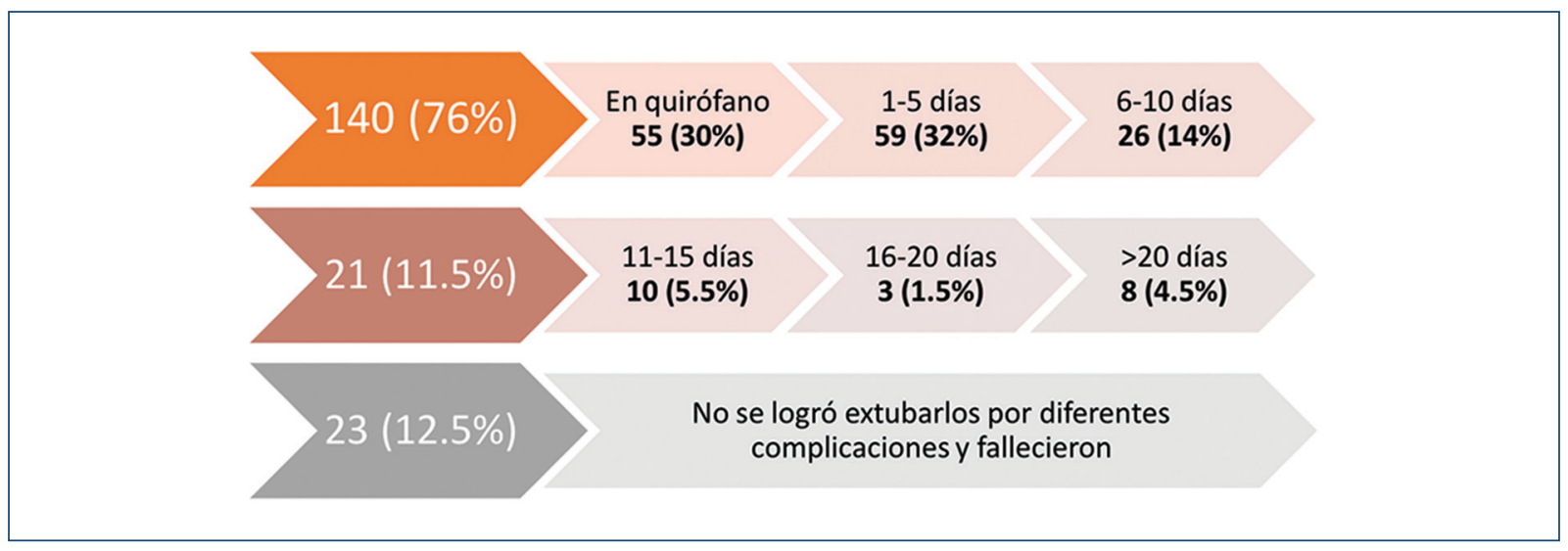

Figura 7. Tiempo de extubación como parámetro de éxito en los pacientes del grupo I.

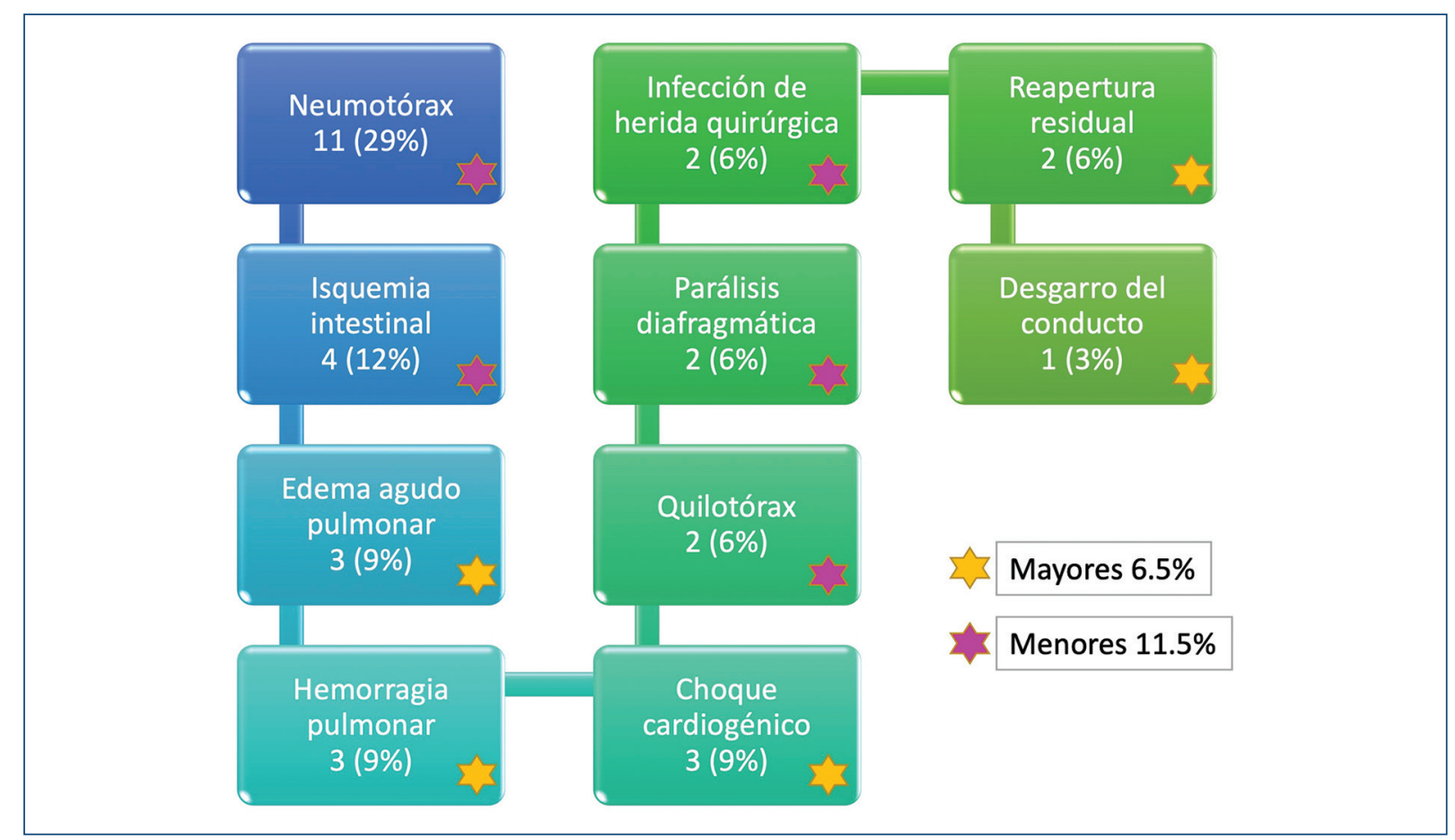

Figura 8. Complicaciones en los pacientes del grupo I.

Ninguno de los pacientes del grupo II fue sometido a cierre farmacológico por la edad. Tenían algún grado de hipertensión pulmonar 12 pacientes $(30 \%)$, de los cuales en $2(17 \%)$ era leve, en 7 (58\%) era moderada y en $3(25 \%)$ era grave. Cursaron sin hipertensión arterial pulmonar 28 pacientes $(70 \%)$.

De los 40 pacientes de nuestra casuística del grupo II, solo $1(2.5 \%)$ tenía una comunicación interventricular como cardiopatía asociada al CAP. En el $82 \%$ se realizó un abordaje quirúrgico extrapleural, en el $12 \%$ transpleural y en el $6 \%$ toracoscópico.
El tiempo de extubación se reporta en la mayoría de los pacientes en quirófano: 34 pacientes (85\%). Del resto, $4(10 \%)$ se extubaron en los primeros 5 días, 1 (2.5\%) a los 6-10 días, 1 (2.5\%) a los $11-15$ días y 1 (2.5\%) no pudo extubarse por presentar complicaciones graves, y falleció (Fig. 12). Las complicaciones del grupo II se reportan en 3 pacientes $(7.5 \%)$, todas ellas menores: 2 pacientes con neumotórax que se resolvió con la colocación de una sonda pleural y 1 paciente con infección o dehiscencia de la herida quirúrgica que se resolvió con curaciones (Fig. 13). En este grupo de 


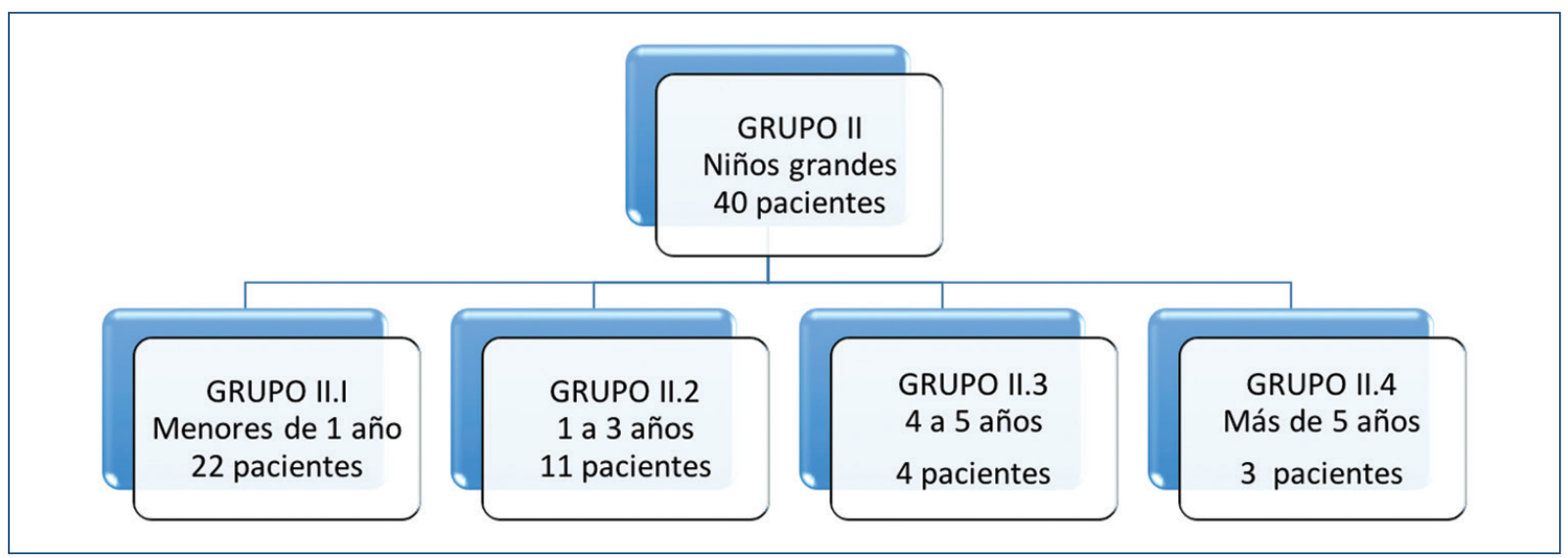

Figura 9. Distribución por edades de los pacientes del grupo II.

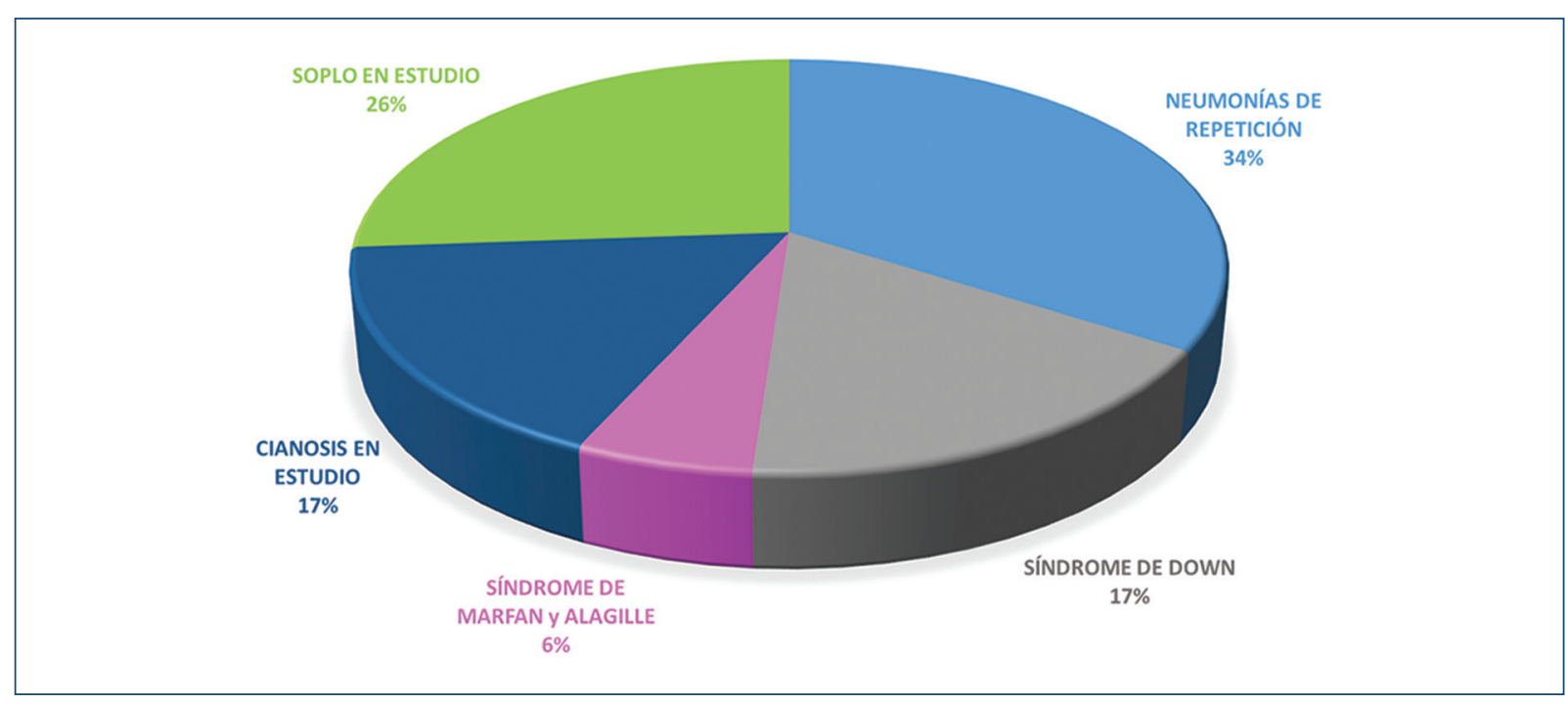

Figura 10. Diagnóstico de ingreso de los pacientes del grupo II.

niños mayores, un paciente falleció por choque séptico que no respondió al manejo hemodinámico intensivo.

\section{Discusión}

En México existen hospitales de segundo y tercer nivel que cuentan con unidades de cuidados intensivos neonatales sin unidades cardiovasculares ${ }^{1-3}$. El CAP es la cardiopatía congénita que con más frecuencia se encuentra en estas unidades y su resolución habitualmente se intenta por cierre farmacológico. Algunos autores señalan una morbimortalidad del $24 \%$ en los pacientes con cierre quirúrgico frente al $13 \%$ en aquellos con cierre farmacológico ${ }^{17-20}$. Sin embargo, un porcentaje variable de RN con CAP-HS no responden al cierre farmacológico o tienen alguna contraindicación formal para intentarlo; es en estos en quienes se debe hacer un cierre quirúrgico, y en muchas de las unidades de cuidados intensivos neonatales no se cuenta con un cirujano cardiovascular pediátrico.

En este estudio no analizamos la evolución de los pacientes con cierre farmacológico, pero cuando este tratamiento falló o no se pudo llevar a cabo por alguna contraindicación, lo tomamos como un parámetro indicativo de cierre quirúrgico, y analizamos únicamente la evolución de los pacientes a los que se les hizo corrección quirúrgica por parte de un cirujano pediatra.

Aunque sabemos que en los niños de más de $2 \mathrm{~kg}$ de peso el intervencionismo para el cierre del CAP es factible, en la mayoría de nuestras unidades que 


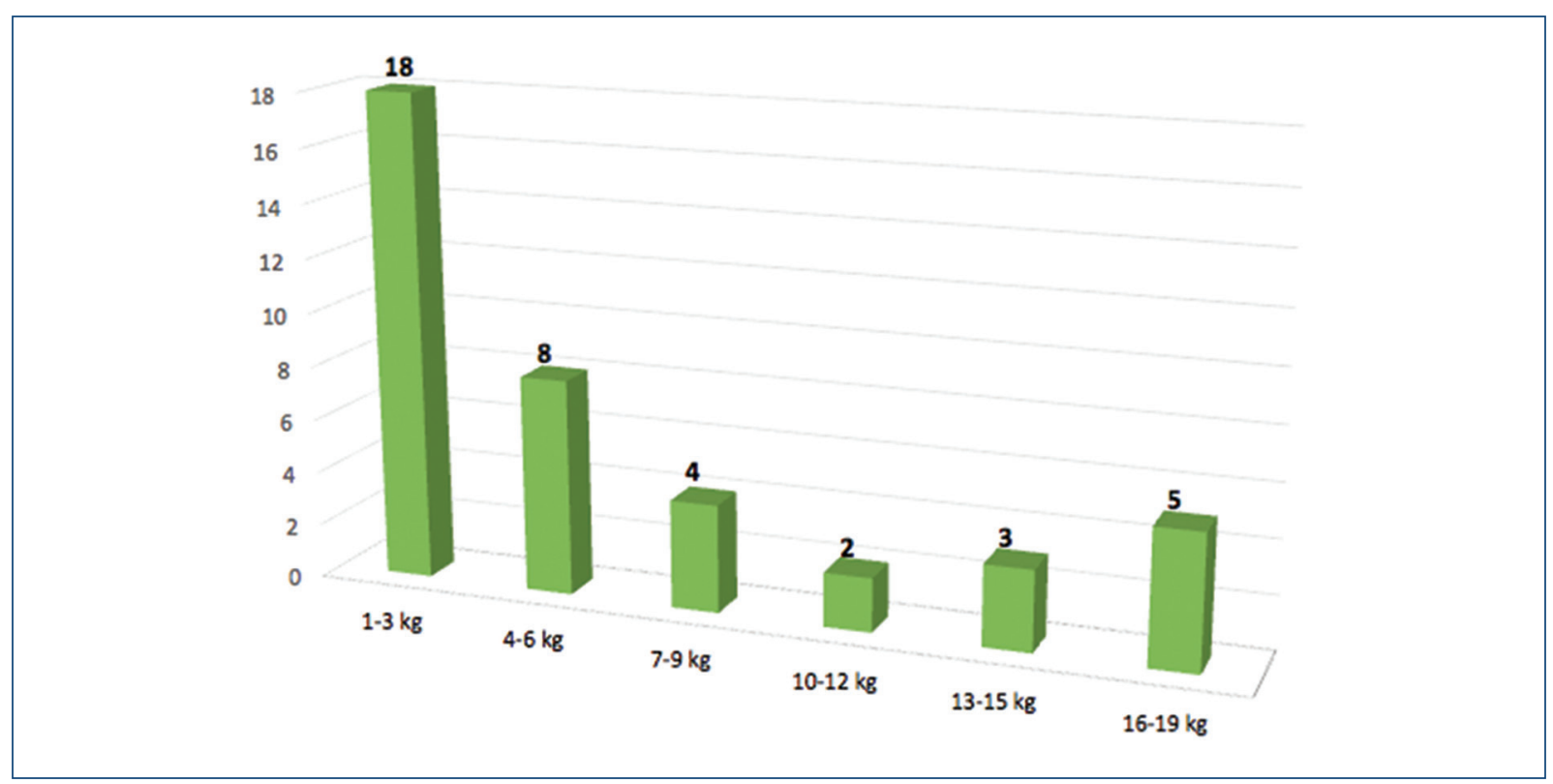

Figura 11. Distribución del peso (de 3 a $19 \mathrm{~kg}$ ) en los pacientes del grupo II.

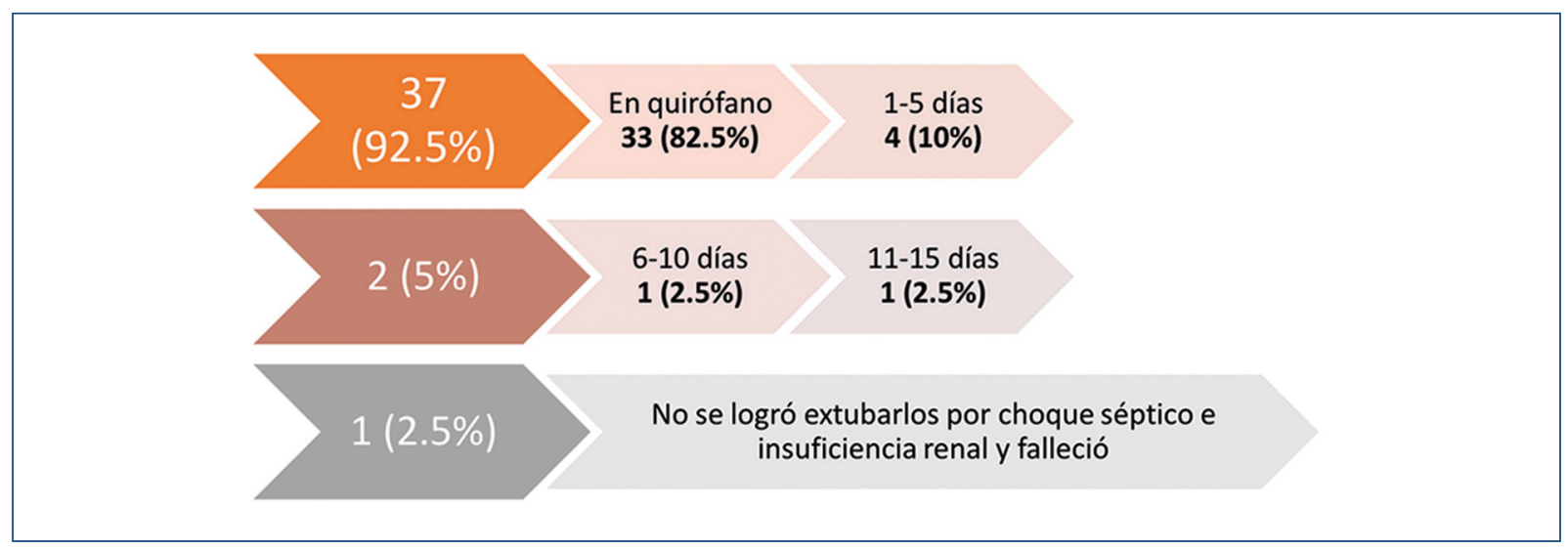

Figura 12. Tiempo de extubación como parámetro de éxito en los pacientes del grupo II.

disponen de terapia intensiva neonatal no se cuenta con sala de hemodinamia e intervencionismo, ni con presupuesto para los dispositivos, y las unidades que sí cuentan con el equipo no darían abasto para atender a todos los RN con CAP-HS.

Las complicaciones reportadas en la literatura atribuidas a la cirugía propia del CAP son intraoperatorias y posoperatorias tardías. Las intraoperatorias incluyen sangrado o hemorragia (4-10\%), desgarro ductal (2$2.5 \%$ ) y lesión del nervio laríngeo recurrente con parálisis de cuerdas vocales (1-8\%). En nuestro estudio solo tuvimos desgarro ductal en un caso, que representa el $0.4 \%$, y se resolvió en quirófano sin complicaciones graves; en nuestro estudio, dicho incidente muestra una incidencia menor que en la literatura revisada. Las complicaciones posoperatorias reportadas en la literatura son neumotórax (1-13\%), quilotórax (1-4\%), infección sistémica $(7-8 \%)$ e infección de la herida (1-2\%); todas presentes y similares en frecuencia a las encontradas en nuestro estudio. Llama la atención que las complicaciones de nuestros casos se asemejan a las de la literatura revisada, y la mayoría son complicaciones menores que fueron resueltas de inmediato ${ }^{21-26}$.

El cierre quirúrgico del CAP en nuestro hospital fue una opción muy útil y con baja morbimortalidad, por lo cual consideramos que es un procedimiento 


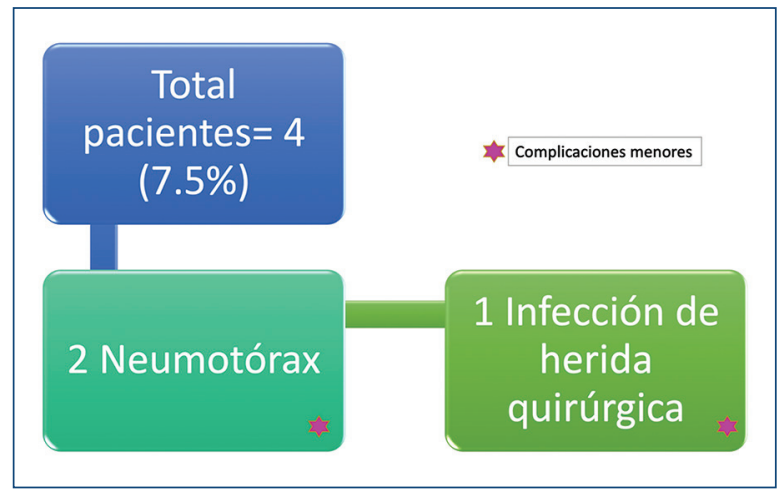

Figura 13. Complicaciones en los pacientes del grupo II.

relativamente sencillo con complicaciones mínimas, que puede llevarse a cabo en un hospital de segundo nivel. Nuestros resultados son muy satisfactorios, con una sobrevida global del $92 \%$.

En nuestra serie, la mortalidad fue del 10.7\% (24 pacientes), siendo todas las muertes no relacionadas con el procedimiento quirúrgico en sí.

Demostramos con este estudio la factibilidad de realizar estas cirugías por un cirujano pediatra, haciendo hincapié en que la mortalidad es equiparable o incluso menor que la reportada en la literatura por centros donde sí tienen cirujanos cardiovasculares pediátricos.

\section{Conclusiones}

En los hospitales pediátricos o generales que no cuenten con un servicio de cirugía cardiovascular pediátrica o de hemodinamia para el cierre percutáneo, el cierre quirúrgico del CAP puede ser llevado a cabo por un cirujano pediatra que tenga experiencia en este tipo de intervenciones. El procedimiento es seguro, fácil de realizar, reproducible y con mínimas complicaciones.

\section{Financiamiento}

Ninguno.

\section{Conflicto de intereses}

Ninguno.

\section{Responsabilidades éticas}

Protección de personas y animales. Los autores declaran que para esta investigación no se han realizado experimentos en seres humanos ni en animales.

Confidencialidad de los datos. Los autores declaran que en este artículo no aparecen datos de pacientes. Los autores declaran que han seguido los protocolos de su centro de trabajo sobre la publicación de datos de pacientes.

Derecho a la privacidad y consentimiento informado. Los autores declaran que en este artículo no aparecen datos de pacientes. Los autores han obtenido el consentimiento informado de los pacientes y/o sujetos referidos en el artículo.

\section{Bibliografía}

1. Hermes-DeSatins ER, Clyman RI. Patent ductus arteriosus: pathophysiology and management. J Perinatol. 2006;26:S14-8.

2. Schneider DJ, Moore JW. Patent ductus arteriosus. Circulation. 2006;114:1873-82

3. Dice JE, Bhatia J . Patent ductus arteriosus: an overview. J Pediatr Pharmacol Ther. 2007:12:138-46.

4. Gallardo-Meza AF, González-Sánchez JM, Piña-Garay MA, Medina-Andrade MA, Cabrera-Rojas H, Lozano-Ruiysánchez A, et al. Experiencia en el cierre quirúrgico de ducto arterioso permeable en una unidad de cuidados intensivos (UCIN) de un hospital de segundo nivel en Guadalajara, Jalisco, México. Bol Med Hosp Infan Mex. 2010;67:127-31.

5. DiMenna L, Laabs C, McCoskey L, Seals A. Management of the neonate with patent ductus arteriosus. J Perinat Neonatal Nurs. 2006;20:333-40.

6. Lee HC, Silverman N, Hinz SR. Diagnosis of patent ductus arteriosus by a neonatologist with compact portable ultrasound machine. J Perinatol. 2007;27:291-6.

7. Hammoud MS, Elsori HA, Hanafi EA, Shalabi AA, Fouda IA, Devarajan LV. Incidence and risk factors associated with the patency of ductus arteriosus on preterm infants with respiratory distress syndrome in Kuwait. Saudi Med J. 2003;24:982-5.

8. Costeloe K, Hennessy E, Gibson AT, Marlow N, Wilkinson AR. The EPIcure study: outcome to discharge from hospital for infants born at the threshold of viability. Pediatrics. 2000;106:659-71.

9. Del Moral T, González-Quintero VH, Claure N, Vanbuskirk S, Bancalari E. Antenatal exposure to magnesium sulfate and the incidence of patent ductus arteriosus in extremely low birth weight infants. J Perinatol. 2007; 27:154-7.

10. Klukopw M, Evans N. Early echocardiographic prediction of symptomatic patent ductus arteriosus in preterm infants undergoing mechanical ventilation. J Pediatr. 1995;127:774-9.

11. Evans N, Malcolm G, Osborn D, Kluckow M. Diagnosis of patent ductus arteriosus in preterm infants. Neoreviews. 2004;45:86-97.

12. Laughon MM, Simmons MA, Bose CL. Patency of ductus arteriosus in the premature infant: is it pathologic? Should it be treated? Curr Opin Pediatr. 2004:16:146-51.

13. Peña-Juárez RA, Medina-Andrade MA, Martínez-González MT, Gallardo-Meza AF, Cortez-Comparan D, Piña-Garay MA. Cierre de conducto arterioso con paracetamol: estudio piloto. Rev Esp Cardiol. 2015; 68:441-51.

14. Bökenkamp R, DeRuiter MC, Van-Munsteren C, Gittenberger-de-Groot $\mathrm{AC}$. Insights into the pathogenesis and genetic background of patency of the ductus arteriosus. Neonatology. 2010;98:6-17.

15. Ohlsson A, Shah PS. Paracetamol (acetaminophen) for patent ductus arteriosus in preterm or low birth weight infants. Cochrane Database Syst Rev. 2018;(4):1-116.

16. Galas MO, Hussain A, Arfi AM. Do we still need the surgeon to close the persistently patent arterial duct? Cardiol Young. 2006;16:522-36.

17. Kabra NS, Schmidt B, Roberts RS, Doyle LW, Papile L, Fanaroff A. Neurosensory impairment after surgical closure of patent ductus arteriosus in extremely low birth weight infants: results from the trial of indomethacin prophylaxis in preterms. J Pediatr. 2007;15:229-34.

18. Koehene PS, Bein G, Alexi-Meskhishvili V, Ewng Y, Burher C, Obladen M. Patent ductus arteriosus in very low birthweight infants; complications of pharmacological and surgical treatment. Perinat Med. 2001;29:327-34.

19. Merrit TA, DiSessa TG, Feldman BH, Kirkpatrick SE, Gluck L, Friedman WF. Closure of patent ductus arteriosus with ligation and indomethacin: a consecutive experience. J Pediatr. 1978;93:639-46. 
20. Mehmet-Yekta O, Sadik Y, Omer E, Nurdan U, Nahide A, Serife-Suna O, et al. Oral paracetamol versus oral ibuprofen in the management of patent ductus arteriosus in preterm infants: a randomized controlled trial. $J$ Pediatr. 2014;164:510-4.

21. Coster DD, Gorton ME, Grooters RK, Thieman KC, Schneider RF, Soltanzadeh $\mathrm{H}$. Surgical closure of the patent ductus arteriosus in the neonatal intensive care unit. Ann Thorac Surg. 1989;48:386-9.

22. Davis JT, Baciewicz FA, Suriyapa S, Vauthy P, Polamreddy R, Barnett B. Vocal cord paralysis in premature infants undergoing ductal closure. Ann Thorac Surg. 1988;46:214-5.
23. Mosalli R, AIFaleh K, Pases $B$. Role of prophylactic surgical ligation of patent ductus arteriosus in extremely low birth weight infants: systematic review and implications for clinical practice. Ann Pediatr Card. 2009;2:120-6.

24. Cassady CD, Kirklin JW. A randomized, controlled trial of very early prophylactic ligation of the ductus arteriosus in babies who weighed 1000 gr or less at birth. N Engl J Med. 1989;320:1511-6.

25. Moin F, Kennedy KA, Moya FR. Risk factors predicting vasopressor use after patent ductus arteriosus ligation. Ann Thorac Surg. 1996:61:814-6.

26. Zbar RI, Chen AH, Behrendt DM, Bell EF, Smith RJ. Incidence of vocal cord paralysis in infants undergoing ligation of patent ductus arteriosus. Ann Thorac Surg. 1996;61:814-6. 